\title{
Mangle; especies nativas de la ciénaga Carrillo del municipio de San Sebastián ${ }^{1}$
}

\section{Mangle; native species of the Carrillo swamp of the municipality of San Sebastián}

DOI: $10.17981 / \mathrm{mod}$.arq.cuc.20.1.2018.10

Artículo. Fecha de recepción: 05/06/2017 Fecha de aceptación: 29/10/2018

\author{
Ricardo Santiago ${ }^{2}$, \\ Dalgis Hernández, Lesbia Ospino, Manuel Ospino y Uriel Hernández \\ IED Alfonso López (Colombia) \\ ridasanva1@yahoo.es
}

Para citar este artículo:

Santiago, R., Hernández, D., Ospino, L., Ospino, M. y Hernández, U. (2018). Mangle; especies nativas de la ciénaga carrillo del municipio de San Sebastián. MODULO ARQUITECTURA-CUC, vol. 20, no. 1, pp. 109-122. DOI: 10.17981/mod.arq.cuc.20.1.2018.10

\section{Resumen}

El propósito del presente estudio fue desarrollar estrategias agrícolas en busca de mejorar el proceso de crecimiento del mangle y otras especies nativas para repoblar la ciénaga del Carrillo, y lograr la implementación de un vivero. Esta propuesta busca contribuir a la conservación del medio ambiente, a través de la educación poblacional, y el manejo adecuado de los recursos naturales. Se trabajó la investigación desde la mirada cualitativa, empleando como técnica de recolección la entrevista estructurada, y la observación participante. La población participante fue la comunidad educativa de San Sebastián-Magdalena. Como conclusiones se infiere que los participantes reconocen la necesidad de repoblar la ciénaga de Carrillo y por tal motivo se encuentran en la fase diagnóstico y adecuación de recursos para la clasificación de especies e implementar el vivero.

Palabras clave: Mangle; especies nativas; ciénaga; estrategias agrícolas; medio ambiente; vivero.

\begin{abstract}
The purpose of the present study it was to develop agricultural strategies in search of improving the growth process of the mangle and other native species to repopulate the Carrillo swamp and achieve the implementation of a nursery. This proposal seeks to contribute to the conservation of the environment, through population education, and the proper management of natural resources. The research was carried out from a qualitative perspective, using the structured interview and the participant observation as a collection technique. The participating population was the educational community of San Sebastián-Magdalena. As conclusions, it is inferred that the participants recognize the need to repopulate the Carrillo swamp and for this reason they are in the diagnostic phase and adaptation of resources for the classification of species and implement the nursery.
\end{abstract}

Keywords: Mangle; native species; swamp; agricultural strategies; environment; nursery.

1 Este artículo ha sido derivado del Programa de Fortalecimiento de la Cultura Ciudadana y Democrática CT+I a través de la IEP apoyada en TIC en el Departamento de Magdalena: CICLÓN. Desarrollado con docentes miembros del grupo de investigación "Mangle, espacio de vida" pertenecientes a la Institución Educativa Departamental Alfonso López.

${ }^{2}$ Líder del grupo de investigación "Mangle, espacio de vida".

- The author; licensee Universidad de la Costa - CUC 


\section{INTRODUCCIÓN}

Al indagar sobre la desaparición paulatina de especies de flora nativa en la ciénaga Carrillo, los estudiantes pudieron analizar la real situación del ecosistema de la región. Por ello, se dieron interrogantes que daban cuenta del interés de conocer un poco más sobre la flora y fauna de la región, y los efectos que ha traído consigo la intervención del hombre en ellas.

Una de las principales preocupaciones fue encontrar soluciones pertinentes, con trabajo mancomunado, que pueda frenar los efectos negativos. con trabajos fuera del aula, para aplicar conocimientos adquiridos como principal motor de motivación y diseño del presente proyecto.

Uno de los principales propósitos es producir el mangle y otras variedades de especies nativas, para poder recuperar dentro del ecosistema su estado original, que en los últimos años ha sido afectado por la relación indebida del hombre con el entorno.

Este proyecto contó con elapoyoyacompañamiento de la comunidad educativa de la IED. Alfonso López y los habitantes de la región; cuyo principal objetivo fue crear un vivero productor de mangle. La naturaleza siempre espera ser reconfortada, de acuerdo al cuidado y protección que se le dé, por lo tanto la construcción del vivero fue permanente, con una superficie de 320 metros cuadrados para una producción mensual de 400 plantas y una producción anual de 4.800 plantas (Casas, 2002). San Martín, Angarita y Mercado (2016) afirman textualmente que,

Fig. 1. Extensión global del manglar.

Fuente: Spalding, Kainuma, \& Collins (2010)

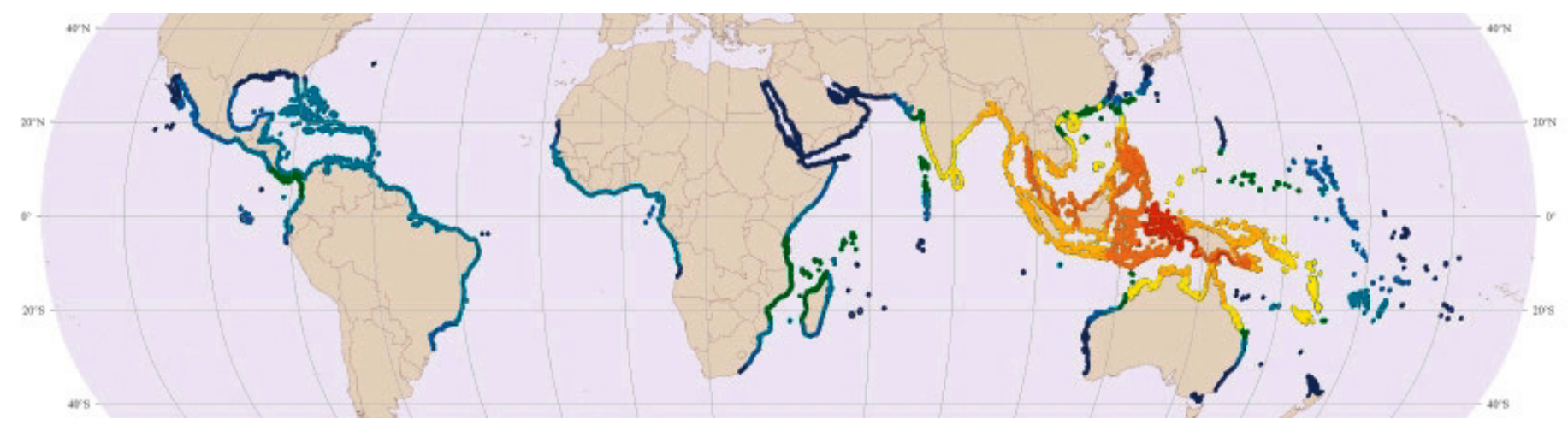



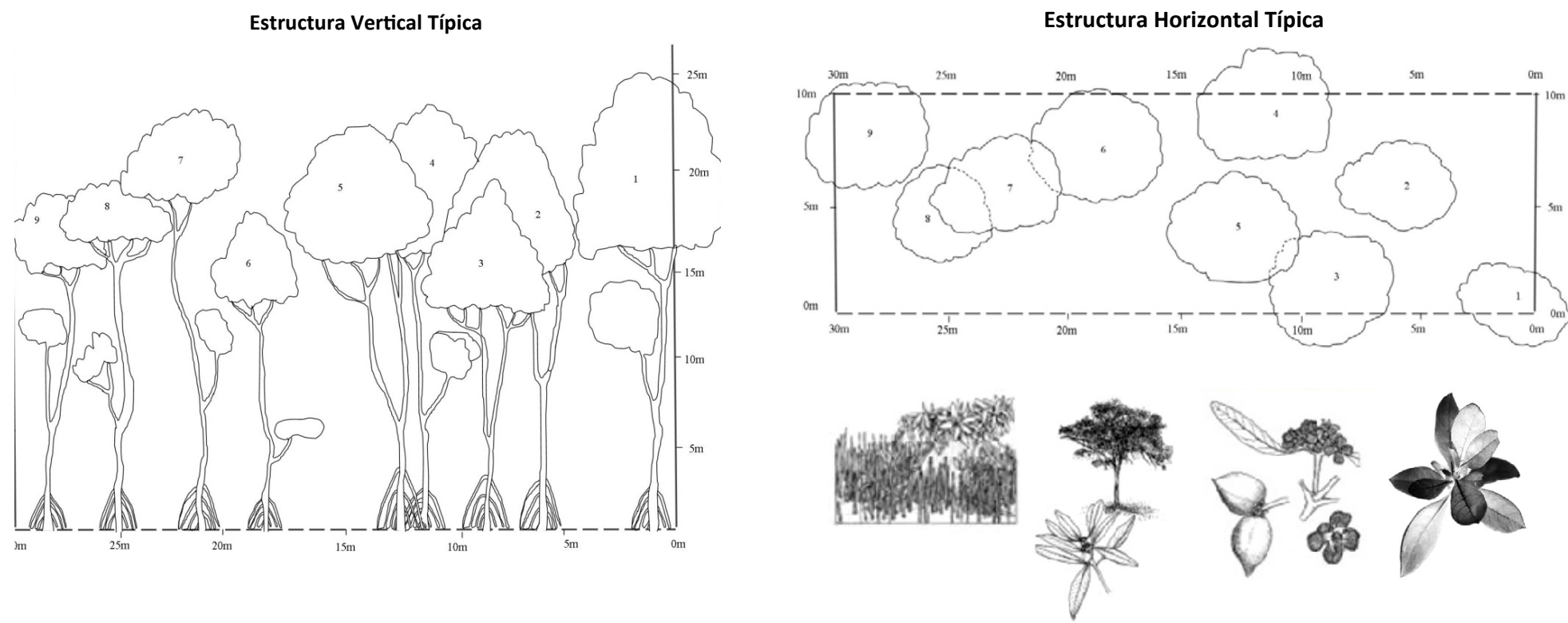

Fig. 2. Bosque de mangle blanco, mangle negro, mangle rojo o mangle boton (Avicennia germinans).

Fuente: Spalding, Kainuma, \& Collins (2010), GIZ (2011).

"en Colombia desde 1960 se ha perdido el $57 \%$ de su cobertura de Manglar, un ecosistema menospreciado y maltratado a lo largo de la historia. Este particular tipo de bosque ocupa un poco más 213.000 hectáreas, según cifras de 2011, de las 501.300 que llegó a tener el país en los años 60" (pp. 2).

El ecosistema marino que tiene una extensión desde la costa continental de Baja California hasta el norte del Perú, evidencia una disminución en gran medida en Panamá, específicamente en el Pacifico Oriental tropical en un 68.2\%, seguida de Ecuador con $62 \%$ por ciento, Colombia con el 57\% y Costa Rica con 40\%. Estos planteamientos son a partir de información recogida entre 1960 y 2011 (Linares, 2016).

Según Linares (2016)

"En el mundo los manglares representan menos del $1 \%$ de los bosques tropicales y subtropicales del planeta y más del $90 \%$ se encuentran en países en desarrollo, donde su tasa de destrucción aumenta a gran escala" (p. 1). 
Las principales causas en la actualidad que atentan contra los ecosistemasde manglar son la agricultura, el desarrollo costero, la acuacultura de camarón, la ganadería y los grupos al margen de la ley, entre otros (López-Angarita, citado por Linares, 2016).

Por su parte en Latinoamérica y el Caribe, el $1,5 \%$ de los manglares se protege de diversas formas. Colombia, con un $28 \%$, posee la más baja cantidad de manglares protegidos, no obstante, en la costa sobrepasa la extensión de los manglares en Panamá. Este país en mención contiene la proporción más alta de los manglares intactos, pero es considerada la más pequeña en la región en cuanto al área que el ecosistema ocupa en su territorio (Linares, 2016).

En las regiones de incidencia de la depresión momposina, brazo de Mompox, se presenta una problemática relacionada con signos de erosión y deforestación asociada, a las largas temporadas de sequias, grandes inundaciones y al impacto de actividades productivas, entre otras, hecho que ha contribuido con la destrucción de los mangles y otras especies nativas de la zona, principalmente en el complejo cenagoso del municipio San Sebastián de Buenavista-Magdalena, desafortunadamente son poca las entidades nacionales y locales que se han preocupado por este caso que nos afecta notoriamente; uno de los sector que más ha contribuido a las grandes erosiones y deforestación es el ganadero en la producción de pastizales (Álvarez, 2003).

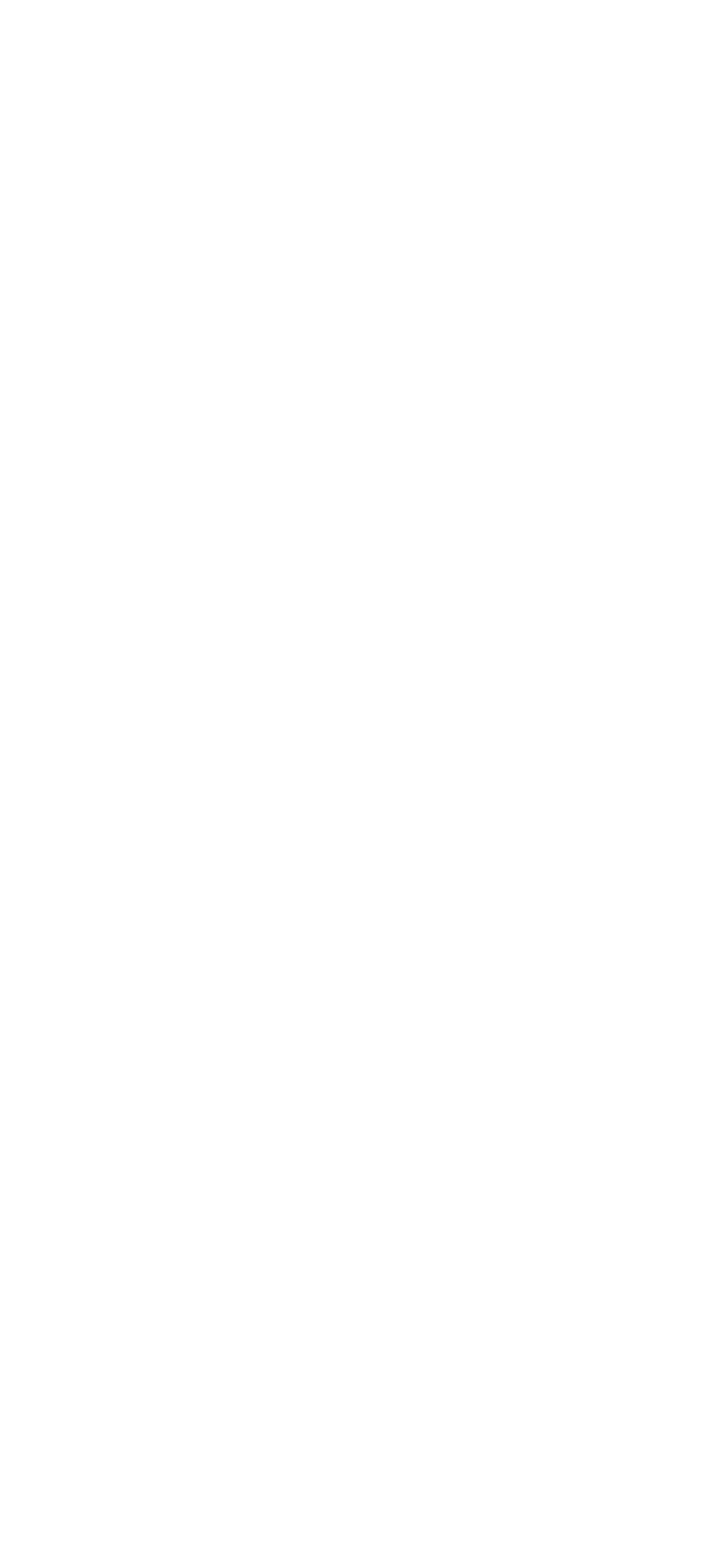

Fig. 3a. Mangle blanco (Laguncularia racemosa), Fuente: González, (2010) 


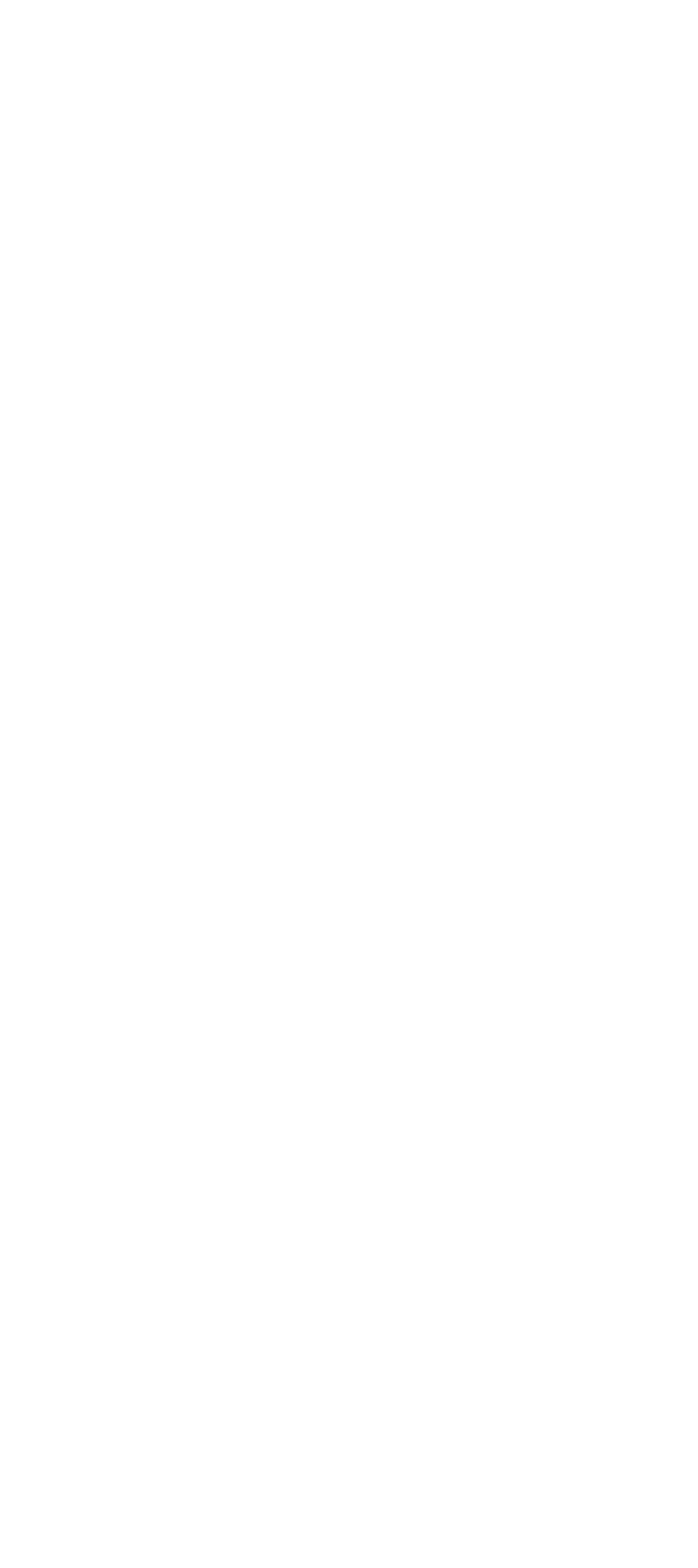

Desde que el hombre buscó refugiarse para protegerse de las condiciones climáticas del medio ambiente, se vio en la necesidad de intervenir el espacio y de acondicionarlo para hacerlo más habitable (Rodríguez y Meza, 2018), sin embargo, esas intervenciones han perjudicado el entorno. En la Institución Educativa Alfonso López de San Sebastián, sede Principal, al indagar sobre la desaparición paulatina de especies de flora nativa en la ciénaga Carrillo, los estudiantes pudieron analizar la real situación del ecosistema de la región. Por ello, surgieron interrogantes que daban cuenta del interés de los jóvenes por conocer un poco más sobre la flora y fauna de la región y los efectos que ha traído consigo la intervención del hombre en ellas.

El mangle hace parte de la flora más representativa de los humedales en el mundo y por ende es pieza fundamental para la conservación de la fauna en los espejos de agua que rodean a nuestro municipio. De ahí la Importancia de este proyecto de investigación que radica principalmente en generar conciencia ambiental a las futuras generaciones y poder contribuir al ecosistema y su entorno natural, que ha sido intervenido por el hombre a través de la deforestación, y se posibilita el devolver al ecosistema parte de su fisonomía original, que en los últimos años se ha visto alterada además de la deforestación, por la intensa sequía e inundaciones, que invaden la región en los últimos años. 


\section{Conceptualización del mangle}

Para Mejía, Molina, Sanjuan, Grijalba y Niño (2014),

"la palabra mangle procede de los indígenas guaraní y significa "árbol retorcido" se refiere principalmente a la especie rhizophora mangle que tiene raíces en forma de zancos que se sumergen en el agua y que asociados a ellos tienen gran diversidad de fauna y flora" (p. 3).

Los manglares son ecosistemas de pantano, que tienen características de árboles leñosos que tienen el nombre de mangles, los cuales se encuentran en litorales tropicales de suelo con características planas y fangosas, y aguas mansas. Los mangles tienen supervivencia en el agua y en la tierra y tienen adaptaciones en sus raíces, hojas y tronco, con los que pueden crecer en terrenos inestables, sin oxígeno e inundados con agua de mar. Según Mejía et al (2014), son los manglares los que ofrecen variadas utilidades sistémicas, los cuales permiten la formación de suelo, considerándose un lugar de hábitat de diversas especies, enriquecen las aguas costeras, brindan protección a la línea de costa, brindan sombrío a las playas y lo más importante proveen la producción de oxígeno.

Fig. 4a. Mangle negro (Avicennia germinans).

Fuente: González, (2010). 
Son los viveros un mecanismo de restauración medioambiental, pues se observa que son varias las razones para que en el entorno se produzcan concentraciones de plántulas bajo la copa de los árboles: una de las más importantes es la desaparición o disminución de la presencia de dispersores; las semillas y frutos, especialmente los más grandes, se depositan bajo la copa de los árboles creando grupos de plántulas entre los que uno o muy pocos logran sobrevivir.

En otros casos, estas concentraciones de plántulas son el resultado de semillas dispersadas en grandes cantidades bajo las perchas de aves o mamíferos. Otra razón para la cual los viveros de plántulas según Flores, Agraz, y Benítez (2006) es que,

"los viveros surgen con la finalidad de producir lo que se denomina cepa siendo indispensable para la siembra, cuando las metodologías implementadas no han sido suficientes. La selección de las especies depende de los diversos caminos de solución con los que se cuenten en el proceso de plantación de reforestación" (2006).

La construcción de viveros forestales es una de las acciones que favorece en gran medida la relación con el medio ambiente, siendo su utilización la que favorece en gran medida la producción y plantación de cultivos los cuales deben ser lugares que deben cumplir con especificaciones en cuanto a la accesibilidad y control del entorno.

Al igual que los materiales sirven en el medio natural u orgánico para ayudar al desarrollo de las especies, pueden encontrarse en las farmacias agrícolas o los conocimientos de personas del medio, (Moreno e Infante, 2016).

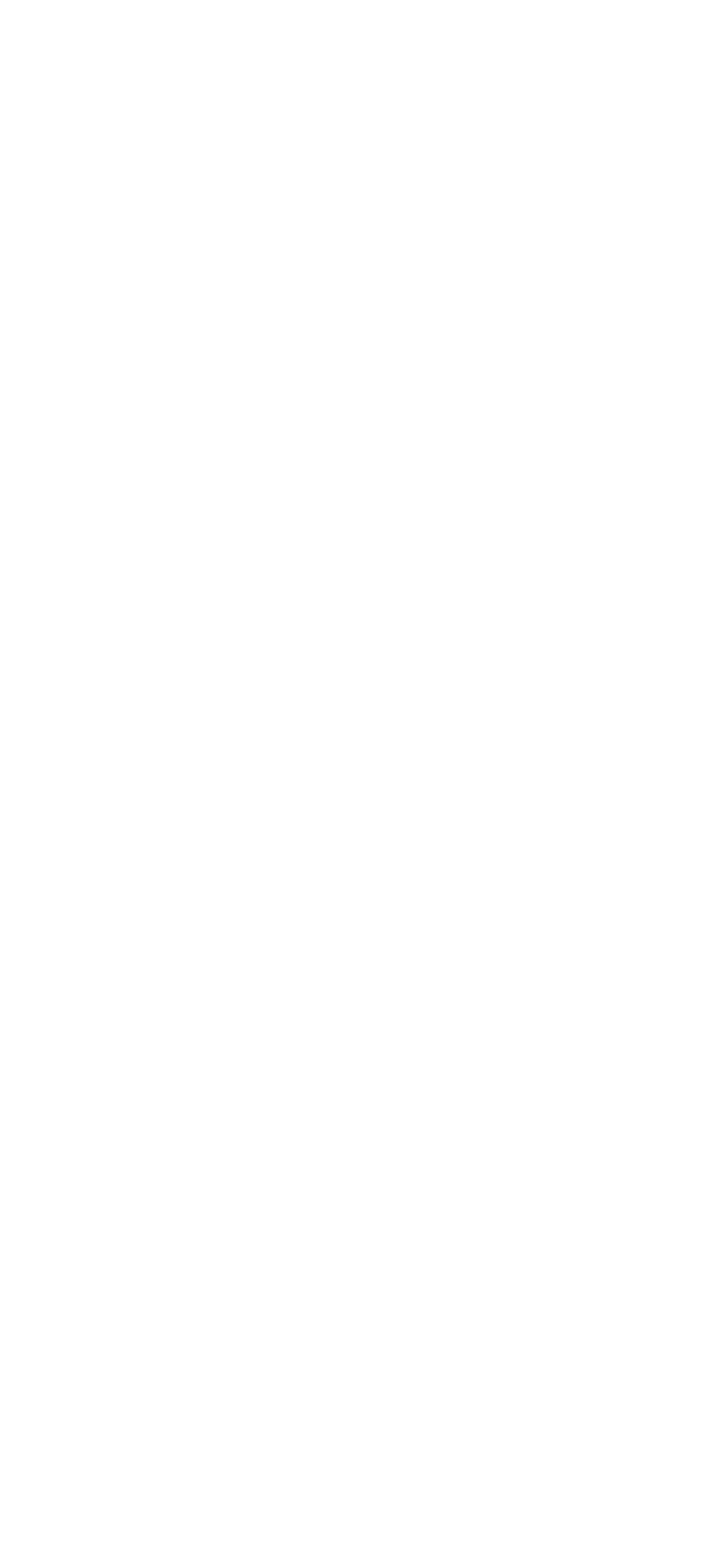

Fig. 5. Mangle rojo (Rhizophora mangle). Fuente: González, (2010) 
Por lo tanto, es de gran importancia lo que los autores exponen, pues contribuye significativamente al propósito de la investigación enmarcado en el crecimiento del mangle y otras especies nativas de la ciénaga Carrillo del municipio de San Sebastián, que servirá como guía en cuanto a las definiciones que contribuyen a la adquisición de herramientas conceptuales y así mismo facilita el cumplimiento de las metas trazadas.

Es importante mencionar que para llevar a cabo el erecimiento del mangle y otras especies nativas en la ciénaga se debe tener en cuenta varios aspectos como; los tipos de mangle, los que se desarrollan más rápido en esta región o suelo, los más fuertes, químicos o elementos naturales que intervienen en el óptimo crecimiento de esta especie y los cuidados que se deben tener para su buen desarrollo (González, 2010).

\section{Metodología}

Este estudio se llevó a cabo desde una mirada cualitativa, siendo ésta la que estudia la realidad en su contexto natural, identificando la naturaleza de la realidad asociada con su comportamiento y sus manifestaciones, además, en su dimensión dinámica busca el cumplimiento de los propósitos de la investigación: categorizar e interpretar datos (Martínez, 2006).

Fig. 6. Mangle boton (Conocarpus erectus).

Fuente: González, (2010).

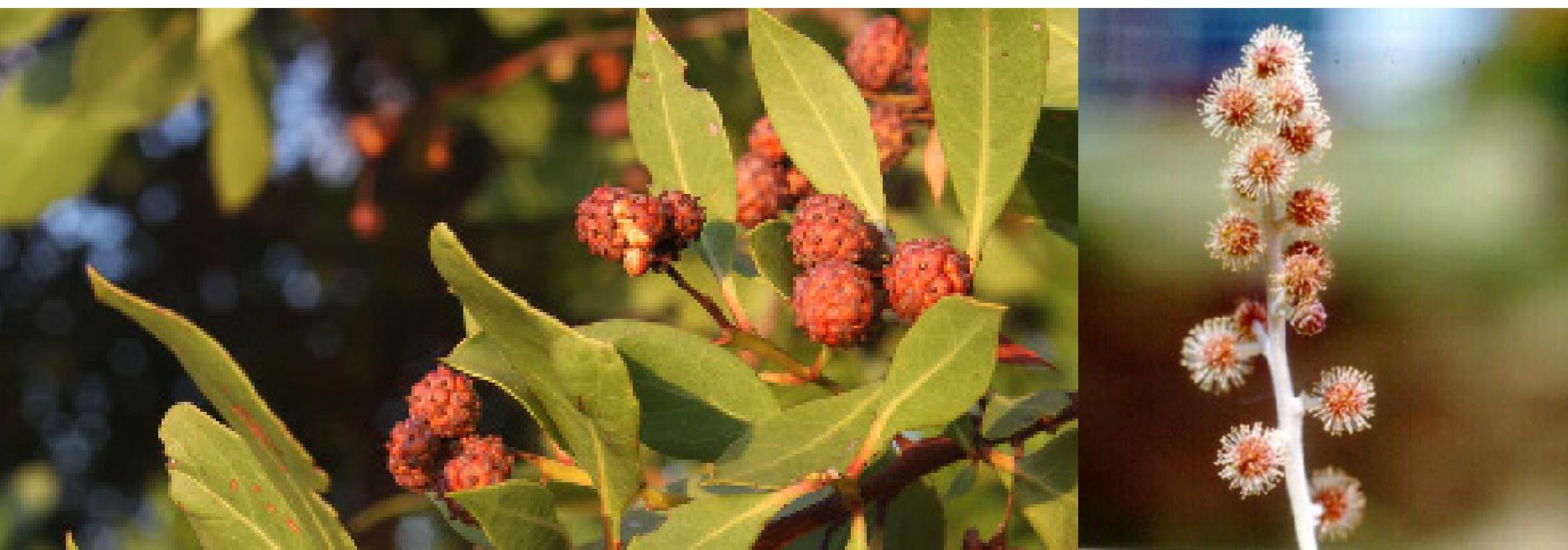




\section{Procedimiento}

Momento (1): Se seleccionan los instrumentos a utilizar.

Momento (2): Se especifican las categorías de estudio.

Momento (3): Se escoge los instrumentos de recolección de información.

Momento (4): Con base a las categorías de estudio: "Crecimiento de mangle" "Especies nativas de la ciénaga"; "Flora y Fauna"; "Medio Ambiente" se aplicaron a los participantes entrevistas, las cuales pretendian describir el fenómeno que se estudia partiendo del nivel de la investigación (no experimental) en donde se observa, y analiza tal y como acontece.
Momento (5): Una vez se tiene los resultados de la entrevista estructurada y la observación registrada se interpretan y comprenden los resultados a la luz de lo que exponen los teóricos que soportan la investigación.

Momento (6): Se relacionan los resultados y se muestran los hallazgos.

\section{Resultados}

A continuación en la tabla 1, se presentan lo hallazgos derivados de los instrumentos aplicados entrevista (voces de los informantes claves) y observación.

\begin{tabular}{|c|c|c|c|}
\hline $\begin{array}{l}\text { Categoría } \\
\text { de estudio }\end{array}$ & $\begin{array}{c}\text { Pregunta } \\
\text { orientadora }\end{array}$ & $\begin{array}{l}\text { Discurso de } \\
\text { los actores }\end{array}$ & $\begin{array}{c}\text { Articulación y } \\
\text { sistematización teórica }\end{array}$ \\
\hline $\begin{array}{l}\text { Crecimiento } \\
\text { del mangle }\end{array}$ & $\begin{array}{l}\text { ¿Puede usted } \\
\text { contarnos } \\
\text { cómo era la } \\
\text { ciénaga Carrillo } \\
\text { anteriormente? }\end{array}$ & $\begin{array}{l}\text { "La ciénaga era un sustento para } \\
\text { pescadores, agricultores y sobre todo } \\
\text { para animales depredadores y aves, } \\
\text { "presentaba gran arborización y no } \\
\text { existía ninguna barrera artificial para } \\
\text { el ingreso", "la ciénaga antes era más } \\
\text { caudalosa, y la única fuente de sustento } \\
\text { más fija que existía aquí en estas zonas", } \\
\text { "antes era más cuidada, más valorada, y } \\
\text { existían más animales", "la ciénaga antes } \\
\text { estaba más cuidada, tenía más peces y } \\
\text { alrededor había más árboles y plantas" }\end{array}$ & $\begin{array}{l}\text { De acuerdo a los hallazgos encontrados } \\
\text { en la entrevista aplicada, se deduce } \\
\text { que existe una conciencia del cambio } \\
\text { que ha sufrido la ciénaga en cuanto a su } \\
\text { estructura; en cuanto a la diversidad de } \\
\text { animales y plantas, pudiendo notarse un } \\
\text { deterioro en la conservación ambiental. } \\
\text { Frente a esto se puede destacar la } \\
\text { importancia de la ciénaga para la } \\
\text { comunidad, el comercio, la alimentación } \\
\text { y para brindar ambiente de armonía. }\end{array}$ \\
\hline
\end{tabular}




\begin{tabular}{|c|c|c|c|}
\hline $\begin{array}{l}\text { Categoría } \\
\text { de estudio }\end{array}$ & $\begin{array}{l}\text { Pregunta } \\
\text { orientadora }\end{array}$ & $\begin{array}{l}\text { Discurso de } \\
\text { los actores }\end{array}$ & $\begin{array}{c}\text { Articulación y } \\
\text { sistematización teórica }\end{array}$ \\
\hline
\end{tabular}

A la luz de lo expresado por los entrevistados, las respuestas brindadas

Especies nativas de la ciénaga cambiado la flora y fauna de la ciénaga Carrillo? con los animales", "por el incremento en la tala de árboles, que hace que cada vez
"Por la contaminación industria que se usted que ha ha convertido en un componente de destrucción para nuestros recursos naturales", "por las inundaciones, que lograr acabar con los árboles, plantas y la contaminación aumente, y las fuentes de oxigeno estén acabándose".

"Si genera un impacto desfavorable,

Medio ambiente

\begin{tabular}{ll}
\hline & ¿Cuántos \\
& mangles o \\
Especies & manglares cree \\
nativas de la & usted que hay \\
ciénaga & actualmente en \\
& la orilla de la \\
& ciénaga?
\end{tabular}

¿Cree usted que el jarillón generará un impacto ambiental en el ecosistema de la ciénaga? ciénaga? porque esa cantidad de tierra tapó gran parte de la flora que rodeaba a la ciénaga", los ganaderos cerraron las vías de acceso" "todo era más complejo, porque se estaba destruyendo el ecosistema", "las afectaciones eran muchas para las personas, animales, etc."

"No tenemos idea, pero no deben ser muchos", "desconocemos de la cantidad exacta, pero hay pocos, porque no ha existido interés por parte de las entidades estatales de apoyar para la recuperación

¿Cómo podríamos acelerar el proceso de crecimiento del mangle y otras especies nativas? apoyar con el trabajo de investigación". de la ciénaga a través de la siembra"

con relación al por qué el cambio generado en la ciénaga, indicaron que las personas lanzan juicios teniendo en cuenta supuestos no científicos, hipótesis de las posibles causas; lo que reafirma la necesidad de hacer investigaciones profundas dentro de las comunidades para esclarecer las situaciones de nuestro entorno. Pero lo que sí está claro es la falta de manglares y flora nativa en estos humedales.

Las respuestas expuestas por las personas de la comunidad hacen notorio que la construcción del Jarillón genera un impacto desfavorable para el ecosistema de la ciénaga Carrillo, y que sin lugar a duda desencadenaron otro tipo de secuelas humanas y medioambientales.

Esta respuesta indica que existe en la comunidad el conocimiento de la escasez del mangle no solo en la ciénaga Carrillo sino en todos los humedales de la región; además también evidencia la poca información precisa de cantidades o estimaciones de esta población.

La respuesta fue la misma para toda la población, manifestando que no tienen ni idea de cómo hacer para "no sabemos cómo hacer, pues reque- que el mangle acelere su proceso riríamos de materiales y revisión de de crecimiento; lo que sí está claro personas expertas en elcaso que puedan es que los involucrados expresan su satisfacción de que se realice esta investigación por cuanto consideran muy necesario la repoblación efectiva de la ciénaga. 


\section{Conclusiones}

Los estudiantes se apropiaron de valores ambientales para el cuidado y conservación del mangle, dada la importancia del mismo, desarrollando destrezas y habilidades en la manipulación de las plántulas de mangle. Lograron compilar la información necesaria para crear el vivero forestal de mangle además de conocer como esta área destinada a la producción de plantas en condiciones controladas y con objetivos definidos, puede brindar posteriormente la oportunidad de generar bienes y servicios, al conviertirse en una alternativa para la recuperación de áreas que se han perdido por múltiples razones.

Se reconoce la necesidad de un vivero para reproducción de mangle y oras especies seleccionadas para la reforestación. En la actualidad la comunidad en general apoya este tipo de propuestas dado que conocen los beneficios del cuidado y la conservación del mangle para la región.

Finalmente, el proyecto contribuye al desarrollo de competencias y habilidades que promueven a la comunidad la sensibilización y apropiación de saberes científicos y ciudadanos que fortalecen destrezas en el área de ciencias naturales y español y, por ende, al mejoramiento de la calidad educativa al volver el entorno socialmente responsables. El proyecto no solo busca la creación de viveros, sino dar inicios al desarrollo de nuevas investigativas, tras el diseño de estrategias agrícolas en busca de mejorar el proceso de crecimiento del mangle y otras especies nativas para en un futuro pensar en repoblar la ciénaga Carrillo con estas.

\section{REFERENCIAS}

Álvarez, R. (2003). Los manglares de Colombia y la recuperación de sus áreas degradadas: revisión bibliográfica y nuevas experiencias. Madera y Bosques, 9(1): 3-25.

Casas, O. (2002). Estado de los manglares en Colombia año 2000. Informe del Estado de los Ambientes Marinos y Costeros en Colombia: Año 2000. Santa Marta: Instituto de Investigaciones Marinas y Costeras INVEMAR.

Flores, F., Agraz, C. y Benítez, D. (2006). Creación y restauración de ecosistemas de manglar: principios básicos. En, P. Moreno-Casasola, R. Presbarbosa y A. Travieso-Bello (Eds.),

Estrategia para el Manejo Costero Integral: El Enfoque Municipal. (1093-1110). Xalapa: Instituto de Ecología A.C., Comisión Nacional de Áreas Naturales Protegidas.

GIZ (2011). Tipos de bosques y contexto del mapeo de la cobertura forestal en centroamérica y república dominicana. Ministerio federal de cooperación económica y desarrollo de alemania. Recuperado de http:// www.reddccadgiz.org/documentos/ doc_1170376601.pdf

González, C. (2010). Manglares. Puerto Rico: Sea Grant. Recuperado de http://academic.uprm.edu/ gonzalezc/HTMLobj-767/encumarmanglar.pdf 
Linares, A. (2016). Manglares en Colombia.

El Tiempo. [Archivo]. Recuperado de http://www.eltiempo.com/archivo/ documento/CMS-16617206

Martínez, M. (2006). La Investigación Cualitativa (Síntesis Conceptual). Revista IIPSI 9(1). 123-146. Recuperado de http://sisbib.unmsm.edu.pe/bvrevistas/ investigacion_psicologia/v09_n1/pdf/ a09v9n1.pdf

Mejía, L., Molina, M., Sanjuan, A., Grijalba, M. y Niño, L. (2014). Bosque de manglar, un ecosistema que debemos cuidar. Cartagena: Universidad Jorge Tadeo Lozano, Instituto Colombiano de Desarrollo Rural. Recuperado de http://observatorioirsb.org/cmsAdmin/uploads/ cartilla-manglar-28pg-(1)_001.pdf

Moreno, P. e Infante, D. (2016). Conociendo los Manglares, las selvas inundables y los humedales herbáceos. Veracruz: Instituto de Ecología A.C. Recuperado de http://www1.inecol.edu.mx/costasustentable/esp/pdfs/Publicaciones/ ManualManglaresYSelvasInundables. pdf

República de Colombia. Ministerio de Ambiente y Desarrollo Sostenible. (2015). Plan Nacional de Restauración. Restauración Ecológica, Rehabilitación y Recuperación de Áreas disturbadas. Minambiente. Recuperado de http://www.minambiente.gov.co/ images/BosquesBiodiversidadyServiciosEcosistemicos/pdf/plan_nacional_ restauracion/PLAN_NACIONAL_DE_ RESTAURACIÓN_2.pdf
Rodríguez, L. y Meza, C. (2018). La construcción sostenible frente a la mitigación del cambio climático. Modulo arquitectura-CUC, 21(1). 9-22. http://dx.doi.org/10.17981/mod.arq. cuc.18.2.2018.01

San Martín, D., Angarita, D. y Mercado, J. (2016). Estructura y composición florística del bosque seco tropical de Sanguaré-Sucre (Colombia).Ciencia en Desarrollo, 7(2). 43-56.

Spalding, M. Kainuma, M. \& Collins, L. (2010). Atlas Mundial de los Manglares. Londres-Washington, D.C.: Organización Internacional de las Maderas Tropicales (OIMT), la Sociedad Internacional de Ecosistemas de Manglar (ISME), la Organización de las Naciones Unidas para la Agricultura y la Alimentación (FA); el Centro Mundial de Vigilancia de la Conservación de la Naturaleza del PNUMA (PNUMA-WCMC), el Programa sobre el Hombre y la Biosfera de la UNESCO (UNESCO$\mathrm{MAB}$ ), el Instituto para el Agua, el Medio Ambiente y la Salud de la Universidad de las Naciones Unidas (UNU-INWEH) y The Nature Conservancy. 